\title{
Differential roles of nitric oxide synthase isozymes in cardiotoxicity and mortality following chronic doxorubicin treatment in mice
}

\author{
Shiwei Deng • Anke Kruger • Albrecht Schmidt • Annegret Metzger • Tiandong Yan • \\ Ute Gödtel-Armbrust • Gerd Hasenfuss • Friedrich Brunner • Leszek Wojnowski
}

Received: 19 November 2008 / Accepted: 23 February 2009 / Published online: 24 March 2009

(C) Springer-Verlag 2009

\begin{abstract}
The roles of individual nitric oxide synthases (NOS) in anthracycline-related cardiotoxicity are not completely understood. We investigated the effects of a chronic treatment with doxorubicin (DOX) on knockouts of the individual NOS isozymes and on transgenic mice with myocardial overexpression of eNOS. Fractional shortening (FS) was reduced in untreated homozygous nNOS and iNOS knockouts as well as in eNOS transgenics. DOXinduced FS decrease in wild-type mice was attenuated only in eNOS knockouts, which were found to overexpress nNOS. No worsening of contractility was observed in DOX-treated eNOS transgenics and iNOS knockouts. Although the surviving DOX-treated nNOS knockouts exhibited no further impairment in contractility, most $(70 \%)$ animals died within 7 weeks after treatment onset. In comparison to untreated wild-type hearts, the nitric oxide (NO) level was lower in hearts from DOX-treated wild-type mice and in all three untreated knockouts. DOX treatment had no effect on NO in the knockouts. These data indicate differential roles of the individual NOS in DOX-induced cardiotoxicity. Protection against DOX effects conferred by
\end{abstract}

S. Deng $\cdot$ A. Kruger $\cdot$ A. Metzger $\cdot$ T. Yan $\cdot$ U. Gödtel-Armbrust $\cdot$ L. Wojnowski $(\bowtie)$

Department of Pharmacology, Mainz University,

Obere Zahlbacher Str. 67,

55131 Mainz, Germany

e-mail:wojnowski@uni-mainz.de

A. Schmidt $\cdot$ G. Hasenfuss

Department of Cardiology and Pneumonology,

University Göttingen,

Göttingen, Germany

F. Brunner

Department of Pharmacology and Toxicology, University of Graz, Graz, Austria
eNOS deletion may be mediated by a compensatory overexpression of nNOS. NOS inhibition-based prevention of anthracycline-induced cardiotoxicity should be eNOSselective, simultaneously avoiding inhibiting nNOS.

Keywords Cardiotoxicity · Mortality .

Nitric oxide synthase $\cdot$ Doxorubicin

\section{Introduction}

Anthracyclines rank among the most effective antineoplastic drugs (Minotti et al. 2004). An unresolved problem associated with anthracycline use is the dose-dependent cardiotoxicity. Years to decades after the chemotherapy, the cardiotoxicity manifests in 5-20\% patients as a congestive heart failure (CHF) due to dilated cardiomyopathy (van Dalen et al. 2006). The implementation of cumulative dosing limits for anthracyclines (e.g., $550 \mathrm{mg} / \mathrm{m}^{2}$ for doxorubicin, DOX) following the seminal study by Von Hoff et al. (1979) did not eliminate cardiotoxicity and CHF. In addition, limiting cumulative anthracycline dose probably diminishes the efficacy of the antineoplastic treatments. Especially problematic is the understandable reluctance of oncologists to administer anthracyclines to cancer patients suffering from cardiac co-morbidities and/or those already treated with anthracycline and requiring further treatment due to tumor progression or metastases. The co-application of anthracycline with the only clinically efficacious cardioprotectant, dexrazoxane, has been limited, due to weakly evidenced but persisting fears of its negative effect on anti-tumor efficacy of anthracyclines.

Inhibition of NOS in the heart has been suggested as an alternative cardioprotective strategy for anthracycline treatments (Fogli et al. 2004). All three NOS isozymes (eNOS, 
iNOS, and nNOS) are expressed in mammalian cardiomyocytes. They reduce anthracycline to semiquinone radicals, which react with oxygen and generate superoxide while anthracycline is restored ("redox cycling") (Garner et al. 1999). Superoxide may react with the physiological NOS product nitric oxide (NO), forming the potent and aggressive cellular oxidant peroxynitrite. The particular sensitivity of the heart to "oxidative stress" generated in response to anthracycline has been attributed to the low reactive oxygen species (ROS) detoxification capacity of cardiomyocytes (Doroshow et al. 1980).

However, the genetic and pharmacological modulation of cardiac NOS activity has led to conflicting results. DOX potentiated mitochondrial toxicity (Chaiswing et al. 2005) and cardiac injury (Cole et al. 2006) in iNOS knockouts and induced cardiac iNOS expression in wild-type animals (Weinstein et al. 2000). This contrasts with a partial protection against DOX-induced heart damage (although not against mortality) conferred by iNOS deletion in another study (Pacher et al. 2003). The disruption of the quantitatively dominant cardiac NOS isozyme, eNOS, protected against DOX-induced heart damage and mortality (Neilan et al. 2007), whereas the unselective NOS inhibitor nitro-Larginine methyl ester (L-NAME) increased mortality associated with DOX treatment (Pacher et al. 2003). Even more confusing are the results of biochemical studies: DOXinduced eNOS transcription accompanied by increased NO production (Kalivendi et al. 2001) contrasts with eNOS activation resulting in augmented superoxide and diminished NO (Vasquez-Vivar et al. 1997) or with NO depletion due to a direct inhibition of eNOS by DOX (Duquaine et al. 2003). Opposing effects of anthracyclines on nNOS activity have been observed in rat brain preparations (Joshi et al. 1996; Luo and Vincent 1994), whereas the role of this isozyme in the cardiac response to anthracycline has not been investigated. Some of these discrepancies likely reflect the considerable analytical challenges associated with cardiac phenotype, as well as with NO and ROS measurements.

Using NOS knockouts and transgenic mice, we investigated the importance of the individual NOS isozymes to chronic anthracycline treatment consisting of four low $(3 \mathrm{mg} / \mathrm{kg}$ ) doses of DOX given over a period of 4 weeks. Such a dosing resembles typical DOX applications in humans and may be a better model of DOX-induced cardiotoxicity than the frequently lethal, single DOX dose of $20 \mathrm{mg} / \mathrm{kg}$ or more applied in most previous experiments with NOS knockouts (Chaiswing et al. 2005; Cole et al. 2006; Neilan et al. 2007; Pacher et al. 2003). Due to the complexity of NOS physiology and analytics, we focused on the clinically relevant parameters of heart failure and on mortality. The results suggest that cardiac protection against DOX can be achieved by a specific inhibition of eNOS, whereas inhibition of nNOS may have a deleterious effect.

\section{Materials and methods}

Animals and experimental procedures

Wild-type C57BL/6 J (B6) mice, nNOS (Huang et al. 1993), iNOS (Laubach et al. 1995), and eNOS (Shesely et al. 1996) knockouts on the same genetic background were purchased from Jackson Lab (Bar Harbor, ME, USA). eNOS transgenic (eNOS-TG) mice overexpressing eNOS exclusively in the cardiac myocytes were described previously (Brunner et al. 2001). DOX (3 mg/kg) was injected intraperitoneally once a week over a period of 4 weeks. The treatment was interrupted for one week between the third and fourth injection for bone marrow recovery, resulting in a cumulative dose of $12 \mathrm{mg} / \mathrm{kg}$. The control groups received $0.9 \%$ saline in parallel. Mortality was recorded daily and the experiments were terminated 4 weeks after the last injection. All of the experimental procedures conformed to Guide for the Care and Use of Laboratory Animals published by the US National Institutes of Health and were approved by the responsible local ethics committee.

\section{Echocardiography}

Four weeks after the last DOX injection, left ventricular function was assessed in a blinded fashion using in vivo echocardiography. Animals were lightly anesthetized with $2.5 \%$ tribromoethanol $(0.01 \mathrm{ml} / \mathrm{g})$ and allowed to breathe spontaneously. A warming pad was used to maintain normothermia. 2D-guided M-mode echoes (30 MHz) were obtained using a VS-VEVO 660/230 High Resolution Imaging System (VisualSonics, Toronto, Canada). Left ventricular end diastolic dimension (LVEDD) and left ventricular end systolic dimension (LVESD) were measured from original tracings; left ventricular fractional shortening (FS) was calculated as (LVEDD-LVESD)/LVEDD and expressed in percent.

High-performance liquid chromatography

At each designated time point, hearts were removed and homogenized in phosphate-buffered saline (PBS). Daunorubicin was added to the homogenate as the internal standard. Proteins were precipitated in a solution containing $\mathrm{ZnSO}_{4}$ and methanol by centrifugation at $15,000 \mathrm{~g}$ for $10 \mathrm{~min}$ in a Biofuge ${ }^{\circledR}$ fresco (Heraeus). One hundred microliters of the resultant supernatant was transferred into borosilicate glass autosampler vials for analysis. Calibration curve was constructed by adding DOX with known concentrations to untreated homogenate. High-performance liquid chromatography (HPLC) was performed, apart from minor modifications, as recently described (Kassner et al. 2008), using a Lichrocart Lichrosphere ${ }^{\circledR}-100$ RP8e column (Merck, Ger- 
many). The mobile phase was prepared by mixing $25 \mathrm{mM}$ ammonium acetate buffer ( $\mathrm{pH}$ 4.6) with acetonitrile $(76: 24, V / V)$. Triethylamine was added to the mixture at the end concentration of $0.05 \%(V / V)$. The fluorescence of DOX was detected at the excitation wavelength of $485 \mathrm{~nm}$ and emission wavelength of $595 \mathrm{~nm}$.

\section{Taqman assay}

Three days after DOX injection, total RNA was extracted from the left ventricle using the Kit PeqGold RNA pure (Peqlab, Erlangen, Germany) and reversely transcribed to cDNA with the High-Capacity cDNA Reverse Transcription Kits (Applied Biosystems). Gene expression levels of three NOS isozymes were measured in triplicate using Taqman assays. For each reaction, $1 \mu \mathrm{l}$ cDNA resulting from reverse transcription of $2 \mu \mathrm{g}$ total RNA was mixed with TaqMan Universal PCR Master Mix (Applied Biosystems) and an appropriate TaqMan Gene Expression Assay from Applied Biosystems (nNOS, Mm00435175_m1; iNOS, Mm00440485_m1; eNOS, Mm00435204_m1). PCR was performed at recommended conditions and mRNA copy numbers were calculated according to standard curves obtained with defined numbers of plasmids containing subcloned individual NOS cDNAs. 18S ribosomal RNA (Hs99999901_s1, Applied Biosystems) was determined in the same samples and the results were used for normalization of NOS expression.

\section{Western blot}

Three days after each injection, three animals from each group were sacrificed and left ventricles were removed and homogenized in buffer containing $50 \mathrm{mM}$ Tris (pH 7.4), $150 \mathrm{mM} \mathrm{NaCl}, 50 \mathrm{mM} \mathrm{NaF}, 1 \mathrm{mM}$ EGTA, $1 \mathrm{mM}$ EDTA, $40 \mathrm{mM} \beta$-glycerophosphate, $10 \%$ glycerin, $1 \%$ Triton X100 , and proteinase inhibitors. Proteins $(50 \mu \mathrm{g})$ were separated by $7.5 \%$ SDS-PAGE gel and subsequently transferred to a PVDF membrane. eNOS isozyme was probed using mouse monoclonal antibody against eNOS (BD Bioscience). Proteins containing 3-nitrated tyrosine were detected with the rabbit anti-mouse polyclonal antibody against 3-nitrotyrosine (3-NT) (Upstate). $\beta$ Tubulin was detected as loading control using mouse monoclonal antibody against $\beta$-tubulin (Sigma). Expression of immunoblotted proteins was visualized using an enhanced chemiluminescence detection kit (Amersham).

\section{Cardiac NO and malondialdehyde measurement}

Cardiac NO and malondialdehyde (MDA) levels were measured 3 days after each injection. Three mice from each group were sacrificed by cervical dislocation and the whole hearts were homogenized in $10 \% \operatorname{PBS}(W / V)$.
After centrifugation through Vivaspin 500 (10,000 MW cutoff) filters (Vivascience AG, Hannover, Germany) to remove the proteins, NO level was assessed in the supernatant as total nitrate and nitrite using the Nitric Oxide Assay Kit from Calbiochem (Cat. No. 482655). MDA level was measured in the supernatants of heart homogenates $\left(3,000 \mathrm{~g}\right.$ for $10 \mathrm{~min}, 4^{\circ} \mathrm{C}$.) using the Lipid Hydroperoxide (LPO) Assay Kit from Calbiochem (Cat. No. 437634) according to the manufacturer's instructions.

\section{Histological study}

After echocardiography, the animals were sacrificed by cervical dislocation; hearts were excised, fixed in buffered $4 \%$ formaldehyde, embedded in paraffin, and cut into 5- $\mu \mathrm{m}$ sections. Cardiac collagen deposition was detected by Azan staining (Liao et al. 2006) according to standard procedures. Histopathological changes were examined using a light microscope independently by two investigators who were blinded regarding the identity of experimental groups.

\section{Statistical analysis}

Results were expressed as mean \pm SE. Comparisons were made between groups with one- or two-way ANOVA followed by post-hoc test. The survival curves were compared by $\log$ rank test. Statistical significance was defined as $P<0.05$.

\section{Results}

Left ventricular dysfunction and reduced cardiac NO level in B6 mice following chronic DOX treatment

Only one B6 mouse died in the DOX treatment group within 60 days after the treatment onset, whereas there was no mortality in untreated mice $(P>0.05, \log$ rank test, $n=10$ per group). Left ventricular function was monitored serially, 2 days, 2 weeks, 4 weeks, and 8 weeks after the last DOX injection. FS gradually decreased in DOX-treated mice from $52 \%$ to $31 \%(P<0.01, n=10$ per group) at 4 weeks after the last injection and remained at this level for another 4 weeks (Fig. 1a). LVEDD increase, indicative of dilated cardiomyopathy characteristic for DOX (Minotti et al. 2004), reached its plateau already 2 weeks after the last DOX injection (Fig. 1b). Therefore, in all subsequent treatment and genotype groups, echocardiography was conducted 4 weeks after the last DOX injection.

Cardiac DOX accumulation was determined by HPLC. After a single dose of $3 \mathrm{mg} / \mathrm{kg}$, DOX was detectable only 
after $24 \mathrm{~h}$ (data not shown). To better delineate the kinetics of cardiac DOX accumulation and elimination, B6 mice were therefore treated with a single injection of $12 \mathrm{mg} / \mathrm{kg}$ DOX and its cardiac accumulation was detected $30 \mathrm{~min}$, $1 \mathrm{~h}, 6 \mathrm{~h}$, and $24 \mathrm{~h}$ after the treatment. We detected no DOX metabolites at these time points. As shown in Fig. 1c, DOX level in heart tissues peaked after $1 \mathrm{~h}$ and rapidly declined to $<20 \%$ of the peak value after $24 \mathrm{~h}$. Therefore, in the following experiments, NO and MDA levels were measured 3 days after each injection, i.e., at the projected end of cardiac DOX elimination. As shown in Fig. 1d, NO level in hearts from B6 mice decreased after the first DOX injection and remained unchanged thereafter ( $n=3$ per group). In contrast, no changes were detected in the marker of oxidative stress MDA (Fig. 1e, $n=3$ per group) nor in the 3-nitrotyrosine (not shown) measured in heart homogenates at the same time points.

Gene expression level of eNOS, iNOS, and nNOS were measured in untreated B6 animals, as well as after the second (2 DOX) and the last (4 DOX) injection. eNOS was the predominant cardiac NOS isozyme in terms of transcript level, followed by iNOS and nNOS (Fig. 2a, $n=4$ per group). Gene expression of nNOS after the last DOX exposure increased by nearly $50 \%$ compared with untreated mice, reaching a borderline significance $(P=0.06)$. This was accompanied by a non-significant trend towards lower levels of eNOS mRNA (Fig. 2a) and a significant one towards lower eNOS protein expression level $(P<0.05$, CON vs. four DOX, Fig. $2 \mathrm{~b}, n=3$ per group). Despite extensive efforts involving various antibodies and experimental conditions, we were unsuccessful in the unequivocal detection of iNOS and nNOS proteins.

Survival, NO, MDA level, and gene expression in NOS knockouts and transgenics following chronic DOX treatment

Out of all treated and untreated eNOS- -- , iNOS $-/-$, and eNOS-TG animals, only one DOX-injected eNOS-/mouse died within 60 days after the treatment onset
Fig. 1 Model of chronic cardiotoxicity employed in this study. a FS monitored in untreated $(C O N)$ and DOX-treated B6 mice 2 days, 2 weeks, 4 weeks, or 8 weeks after the last DOX exposure. $\# P<0.05, * P<0.01$ vs. $\mathrm{CON} ; n=10$ per group. $\mathbf{b}$ LVEDD monitored in $\mathrm{B} 6$ mice at the same time points as in $\mathbf{a}$; $* P<0.01$ vs. CON. c DOX was detected by HPLC in heart tissues from B6 mice $30 \mathrm{~min}, 1 \mathrm{~h}$, $6 \mathrm{~h}$, and $24 \mathrm{~h}$ after a single injection $(12 \mathrm{mg} / \mathrm{kg}) ; n=6$ per group. d NO level measured in heart homogenates from DOXtreated B6 mice 3 days after each injection. ${ }^{*} P<0.05$ vs. $\mathrm{CON} ; n=3$ per group. e MDA level measured in heart homogenates from DOX-treated B6 mice 3 days after each injection; $n=3$ per group
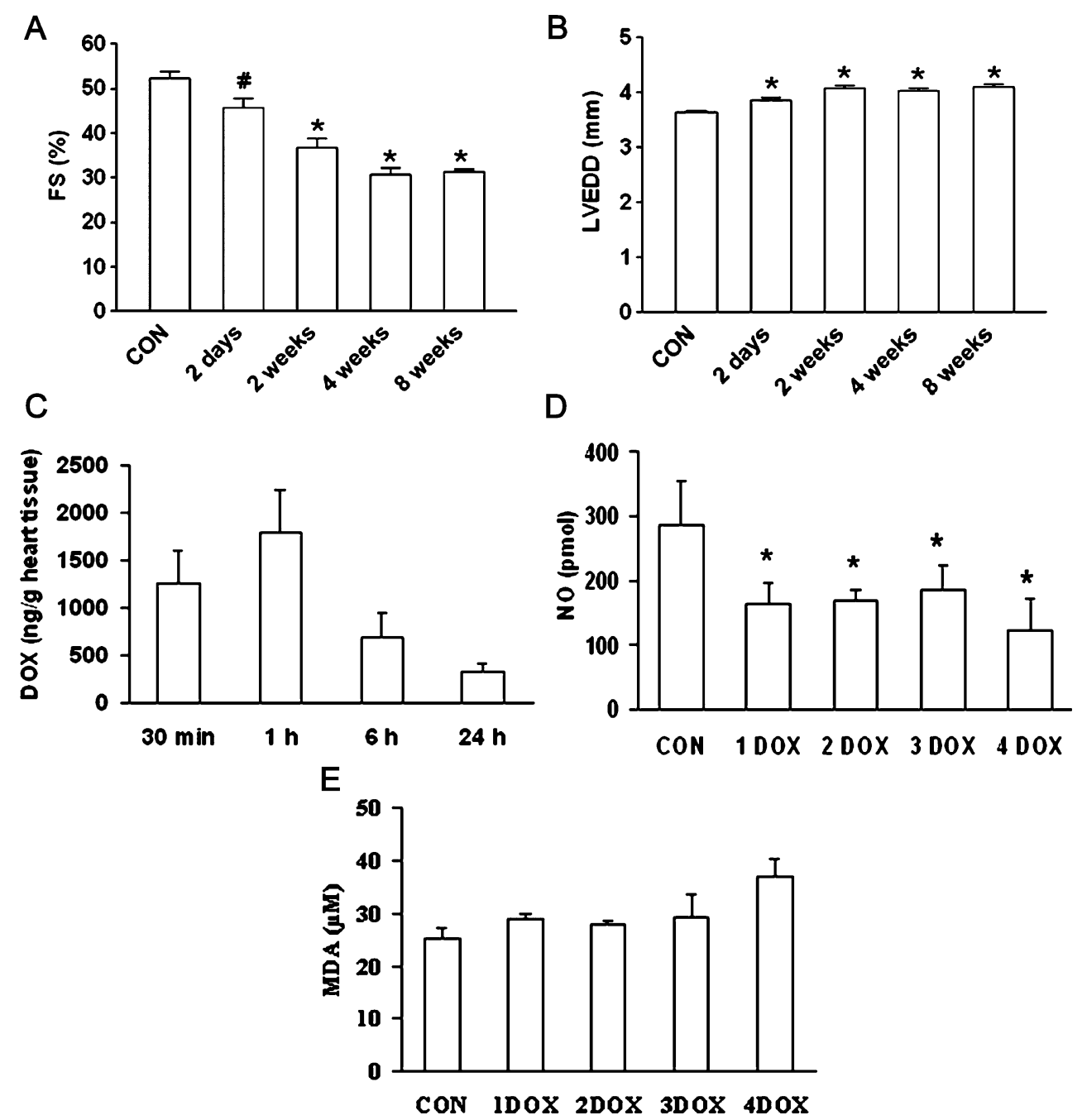


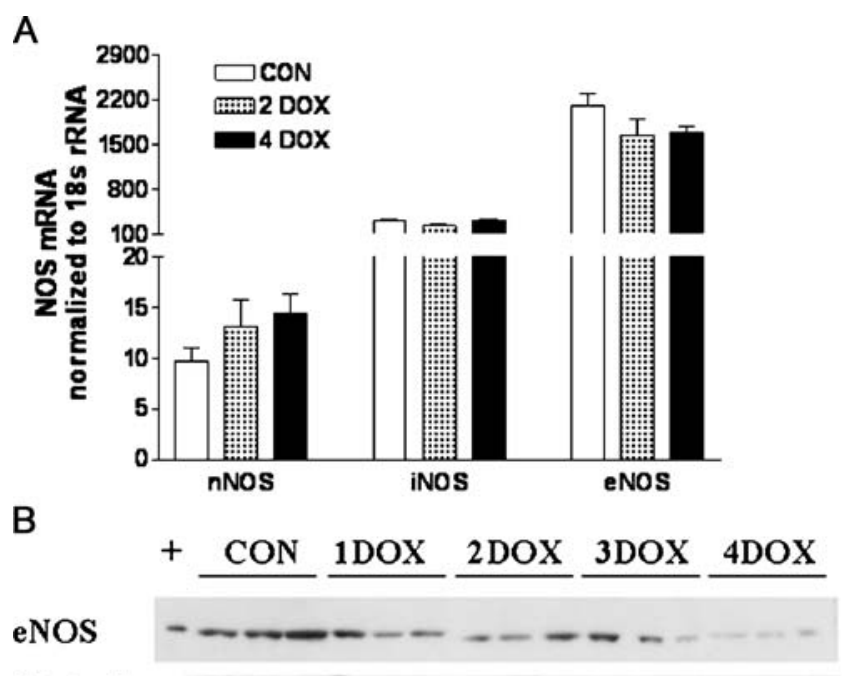

$\beta$-tubulin

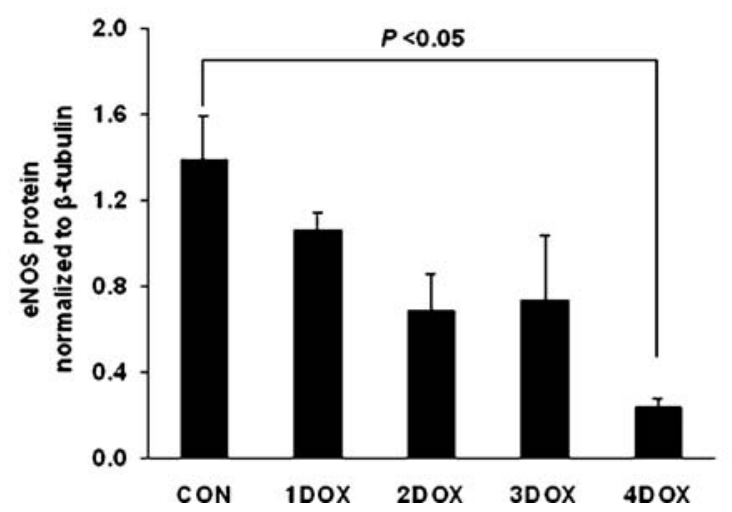

Fig. 2 a Transcript levels of the three NOS isozymes in untreated $(C O N) \mathrm{B} 6$ mice as well as 3 days after the second $(2 \mathrm{DOX})$ and fourth (4 DOX) exposures; $n=4$ per group. b eNOS protein expression in heart homogenates from untreated $(C O N)$ B6 mice as well as 3 days after each injection. Positive control: recombinant eNOS protein. $B-$ tubulin was used as a loading control. The eNOS signals were normalized to $\beta$-tubulin and the ratio between them was used to quantify eNOS protein level

(Fig. 3a, $n=10$ per group). In contrast, 70\% (7/10) of the DOX-treated, but none of the untreated, nNOS- - mice died within the same time period $(P<0.01, \log$ rank test). The nNOS $-/-$ mice mortality was particularly pronounced after the last DOX injection (Fig. 3a).

NO level was much lower in heart homogenates of all NOS knockouts as compared to B6 mice (Fig. $3 \mathrm{~b}, n=3$ per group). A DOX treatment-associated NO decrease was observed exclusively in B6 mice (Figs. 1d and 3b). Neither MDA level nor 3-NT changed in any of the knockouts after DOX exposure (data not shown).

A 2.5-fold overexpression of nNOS gene transcripts was found in untreated eNOS knockouts compared to untreated B6 mice $(P<0.05, n=4$ per group). DOX treatment had no effect on nNOS gene expression in eNOS $-/-$ mice (Fig. 3c).
Alterations of cardiac function and histopathology in NOS knockouts and transgenics following chronic DOX treatment

Left ventricular function and diameter were evaluated in all treatment and genotype groups 4 weeks after the last DOX exposure (Fig. 4, $n=3$ in DOX-treated nNOS knockouts and $n=6$ in other groups). Compared to untreated B6 mice, untreated iNOS-/-, eNOS-TG, and $\mathrm{nNOS}-/-$ mice showed reduced baseline FS values. Significant changes in FS (a decrease, Fig. 4a) and LVEDD (an increase, Fig. 4b) upon DOX treatment were observed exclusively in B6 mice. The representative images from echocardiography measurements are displayed in Fig. 4c. In short, untreated eNOS-/- mice had normal cardiac function and were less sensitive to DOX treatment compared to B6 mice. The contractility in eNOSTG, iNOS-/-, and nNOS-/- mice did not get worse following DOX exposure.

Cardiac collagen deposition, reflecting heart fibrosis, was detected in heart sections by histological staining. The representative micrographs are shown in Fig 5. DOX treatment resulted in pronounced perivascular fibrosis. Untreated iNOS or nNOS knockouts showed higher collagen content compared with untreated B6, without changes between DOX-treated and -untreated animals within the same genotype. These observations were consistent with the functional data obtained from echocardiographic measurements.

\section{Discussion}

Our data indicate highly differential roles of the individual NOS isozymes both in normal heart function and following a chronic DOX treatment. eNOS knockouts exhibit increased contractility in response to $\beta$-adrenergic stimulation, although basal contractility is the same as in wild-type animals (Mungrue et al. 2002), as also observed in our study. The reduced FS values in eNOS-TG mice reported here are consistent with previous observations of diminished cardiomyocyte contractility in this mouse strain (Brunner et al. 2001), attributed to chronically elevated NO generation due to eNOS overexpression (Mungrue et al. 2002). The reduced FS values in nNOS knockouts are in agreement with the reduced cardiomyocyte contractility previously described in this mouse strain in response to $\beta$ adrenergic stimulation (Barouch et al. 2002). The FS reduction in untreated iNOS knockouts is more difficult to explain, since the intracellular localization and physiological role of this NOS isozyme in the heart are less well understood. Although iNOS is frequently and incorrectly assumed to be expressed in the heart only under pathological conditions, it has been previously detected in normal 
Fig. 3 The effect of NOS status on survival and cardiotoxicity. a Survival curves of DOXtreated and untreated $(\mathrm{CON})$ eNOS-l-, iNOS-l-, nNOS-land eNOS-TG mice. Arrows indicate the time when DOX was injected. $P<0.05$ for DOX-treated nNOS-/- vs. CON nNOS- - , log rank test, $n=10$ per group. b Cardiac NO levels measured 3 days after the first DOX exposure, $n=3$ per group. c nNOS transcripts in untreated B6 and eNOS $-/-$ mice as well as DOX-treated eNOS-/- mice 3 days after the first, second, and third DOX exposure, $n=4$ per group

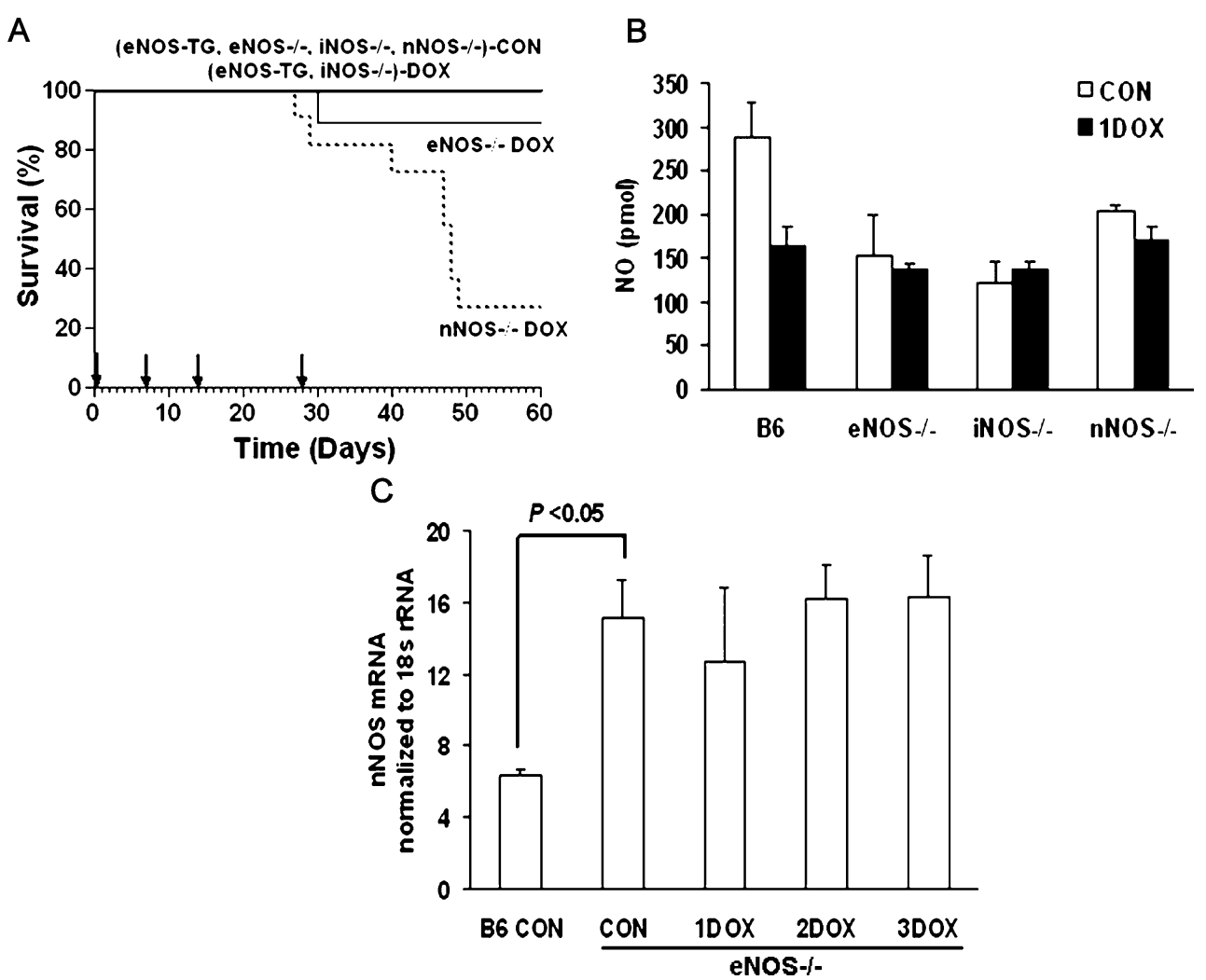

ferret ventricles (Brahmajothi and Campbell 1999). Our detection of iNOS mRNA expression in hearts of untreated wild-type mice, taken together with the presence of a cardiac phenotype (reduced FS) in iNOS knockouts, likewise points to a physiological role of iNOS in this organ. In summary, our data from untreated mice with altered NOS expression support the growing evidence of cardiomyocyte contractility undergoing a complex regulation by the individual NOS isozymes. Importantly, NOSgenotype contractility changes in untreated animals were not accompanied by heart dilatation, which was observed only in wild-type mice following DOX treatment.

The expression status of the individual NOS isozymes had very different effects on heart function in DOX-treated animals, ranging from protection (eNOS-/-, iNOS-/-, eNOS-TG) to excessive mortality (nNOS-/-). Although these differences are valid only for the specified follow-up period, the present study is one of the longest on the subject of DOX-induced cardiotoxicity. The protective effect of iNOS deletion against DOX is in agreement with Pacher et al. (2003), but in contrast to the results by another group (Chaiswing et al. 2005; Cole et al. 2006). In contrast to our work, all these previous studies used much higher sublethal doses of DOX and very short follow-ups (several days), which limit both their utility as a model of anthracyclineinduced cardiotoxicity in humans and the comparability to our results. The lower single DOX doses administered in our study may explain the absence of iNOS induction in wild-type mice, in contrast to that of Weinstein et al. (2000). The same applies to the discrepancy between our data and the recently reported heart failure and mortality within days after a single sublethal DOX dose in mice overexpressing eNOS in cardiomyocytes (Neilan et al. 2007). In our study, a similar eNOS overexpressing mouse strain (eNOS-TG) shows neither a deterioration of FS values nor mortality following a chronic DOX treatment. In contrast, eNOS deletion had a clearly protective effect against DOX, which was in agreement with the study of Neilan et al. (2007), whereas the deletion of nNOS was lethal to most DOX-treated animals. eNOS knockouts develop hypertension (Mungrue et al. 2002) which, however, did not aggravate the response to DOX. While extracardiac effects of DOX on nNOS (as well as on all other) knockouts cannot be excluded, the mice died suddenly, in the absence of any other signs of sickness. Nevertheless, additional studies are still required to understand the molecular mechanisms underlying the striking mortality in nNOS knockouts. Interestingly, increased heart rate, as described in nNOS knockouts, has been associated with increased mortality in humans with heart failure (Mungrue et al. 2002).

Phenotypic differences of this magnitude were unexpected, considering the biochemical similarities among the NOS isozymes in respect of NO production and anthracy- 

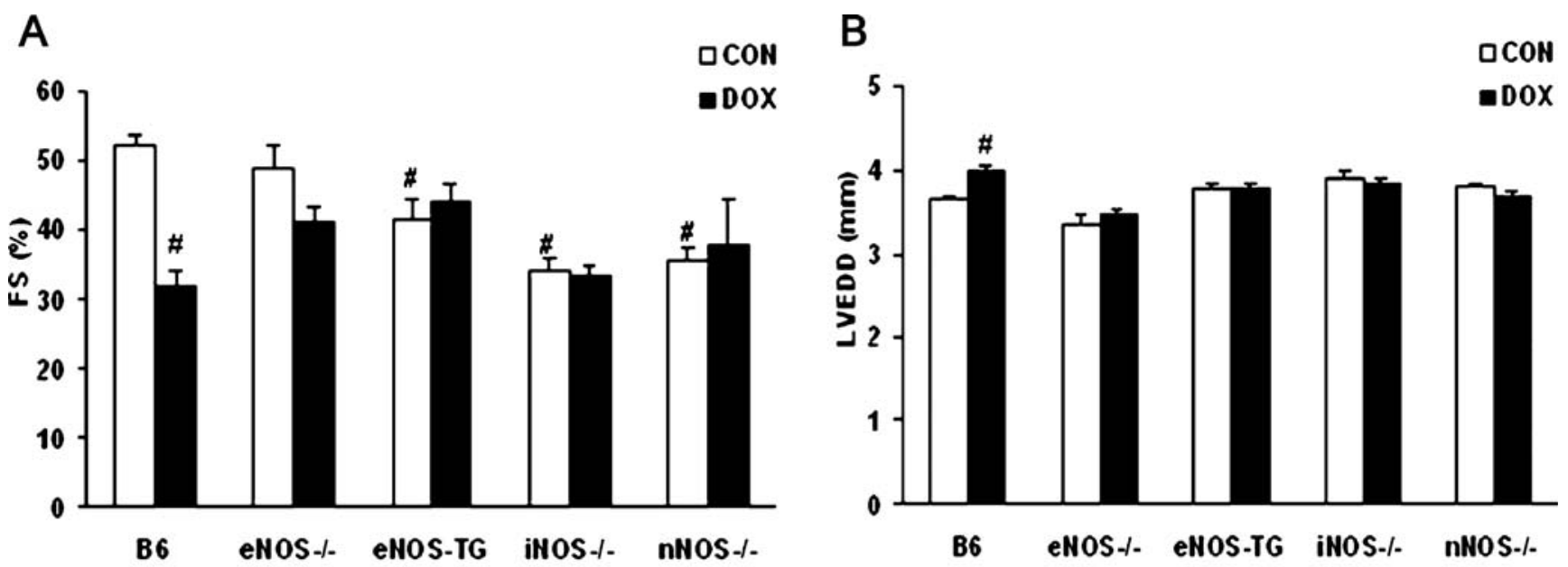

C

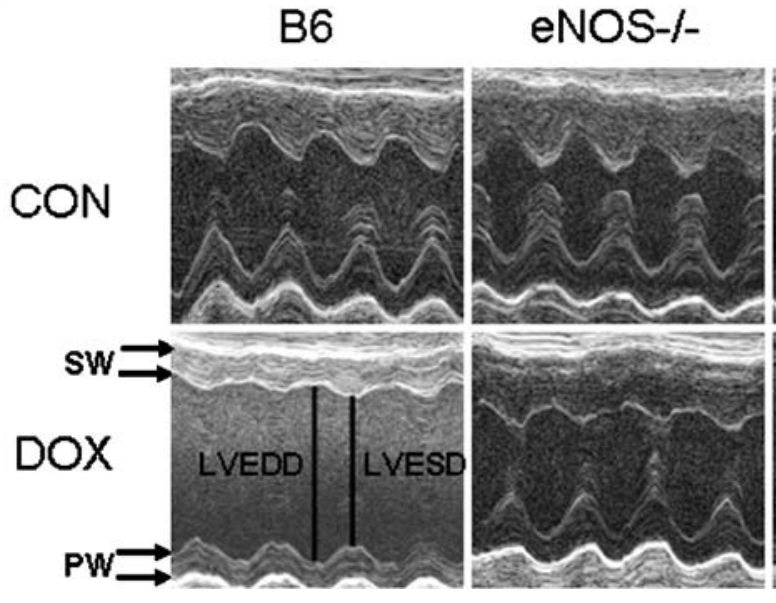

Fig. 4 Alterations of cardiac function in NOS knockouts and transgenics following DOX treatment. a FS and b LVEDD values 4 weeks after the last DOX exposure, $\# P<0.05$ vs. untreated $(C O N)$

B6, $n=3$ in DOX-treated nNOS knockouts and $n=6$ in other groups. c Representative images from echocardiography study. $S W$ septal wall thickness, $P W$ posterior wall thickness

cline "redox cycling" (Garner et al. 1999). In fact, our data suggest that DOX effects may be, at least in part, independent from NO and ROS. Indeed, despite the very different effects on heart function and survival, all three knockouts display similar diminished NO levels, which remain unaffected by DOX. DOX-induced NO decrease occurs only in wild-type mice, probably due to inhibition (Duquaine et al. 2003) rather than decreased expression of eNOS, since the latter type has a much slower kinetics (compare Figs. 1c and 2b). Alternatively, NO effects relevant to DOX response may take place in subcellular compartments too small to be detected by methods applied in our work.

ROS has been suggested to be involved in anthracyclineinduced cardiotoxicity, in part via decreased mRNA and protein level of manganese superoxide dismutase (MnSOD) (Li et al. 2000; Li and Singal 2000). Likewise, less susceptibility to cardiotoxicity has been observed in DOX- treated MnSOD-overexpressing mice (Cole et al. 2006; Yen et al. 1996). We failed to detect enhanced oxidative stress in wild-type mice following DOX exposure which clearly results in a deterioration of heart function. Admittedly, this failure may have resulted from the limited sensitivities of the methods used or from the relatively late time point of its assessment. On the other hand, it is in agreement with the persisting doubts regarding the importance of oxidative stress in the pathogenesis of anthracycline-induced cardiotoxicity. Indeed, it has been noted that ROS following anthracycline exposure is usually detectable only at supraclinical or even sublethal anthracycline doses (Gewirtz 1999). Furthermore, most ROS inhibitors or scavengers failed to prevent anthracycline-induced cardiotoxicity in clinical trials (van Dalen et al. 2005). The only exception, the iron chelator dexrazoxane, has been recently suggested to exert its protective effect on cardiomyocytes via degradation of the topoisomerase II beta rather than ROS inhibition (Lyu 
Fig. 5 Representative micrographs showing perivascular cardiac collagen deposition detected with azan staining (blue color, arrows). Scale bar $=100 \mu \mathrm{M}$

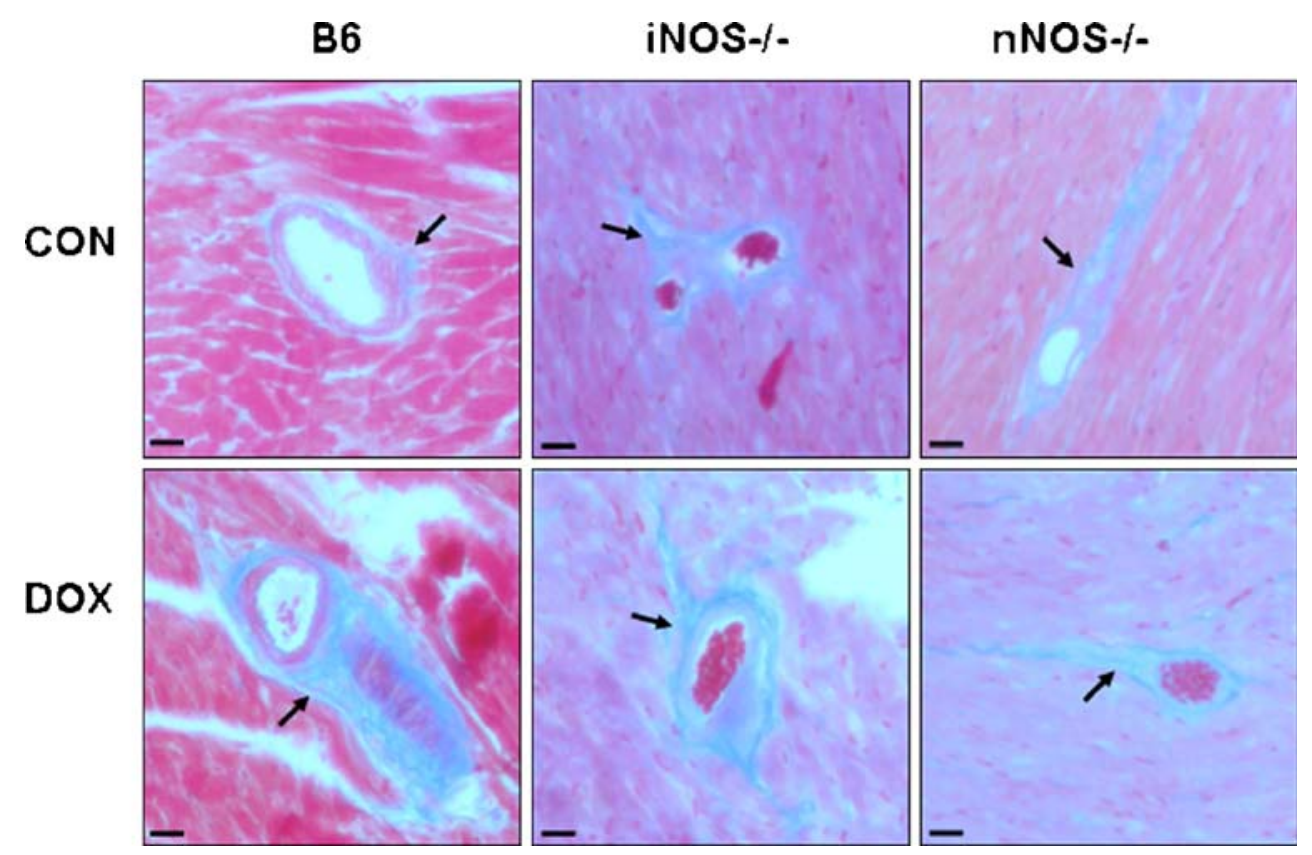

et al. 2007). Furthermore, eNOS and nNOS disruptions had very different effects on heart function and survival following DOX treatment, although both would be expected to reduce ROS production, consistent with the capability of all NOS isozymes to redox cycle anthracycline (Garner et al. 1999).

Instead, the protective effect of eNOS deletion against DOX may be primarily related to the overexpression of nNOS found in this study in hearts of eNOS knockouts. nNOS overexpression has previously been reported in other organs of eNOS-/- mice (Sanz et al. 2001) and may represent a compensatory response. The gradual decrease in eNOS expression found here in DOX-treated wild-type animals may represent a protective response against DOX, which remains insufficient, since it is not accompanied by significant changes in nNOS expression. Conversely, nNOS downregulation or deletion would be expected to be harmful, which is, indeed, the case. In short, the protective effect of eNOS deletion against DOX may be mediated by compensatory overexpression of nNOS. Accordingly, nNOS would be a downstream effector of eNOS, indispensable to protection against DOX, as evidenced by the high mortality in nNOS-/- mice. An indispensable and dominant role of nNOS downstream of eNOS has been postulated for the beta-adrenergic response, based on phenotypical similarities of nNOS and double eNOS/nNOS knockouts (Barouch et al. 2002). The disparate, protective, and harmful effects of nNOS and eNOS deletions are reminiscent of their opposing effects on cardiomyocyte contractility, which are mediated by calcium. Disturbances of calcium homeostasis have been implicated in anthracycline-induced cardiotoxicity (Gewirtz 1999). Altogether, the disparate cardiac effects of the individual NOS may be related to disturbances in the subcellular calcium homeostasis rather than to NO or ROS. Additionally, as nNOS has been reported to regulate blood flow (Talman and Nitschke Dragon 2007), the compensatory increase of nNOS in eNOS-/- mice may have led to coronary vasodilation and improved cardiac microcirculation, thus contributing to the protective effect against DOX-induced cardiotoxicity.

Taken together, the phenotypes of DOX-treated eNOS and nNOS knockouts suggest an explanation for the lethal effect of the unselective NOS inhibitor L-NAME, when given to mice together with DOX (Pacher et al. 2003). It appears likely that this lethality was caused by the inhibition of the indispensable function of nNOS discussed above. Even more importantly, inhibition of nNOS may have been responsible for the similarly unexplained reduced cardiac output, heart failure, and increased mortality in sepsis patients treated with another unspecific NOS inhibitor, L-NMMA (Lopez et al. 2004).

In agreement with Neilan et al. (2007), our data suggest that any NOS modulation-based prevention of anthracyclineinduced cardiotoxicity should aim at a maximally specific inhibition of eNOS. Selective nNOS upregulation may be an alternative, but this remains to be demonstrated using an appropriate mouse model. Most critical is the avoidance of nNOS inhibition, which is associated with the increased mortality in our study and may have increased mortality in previous clinical (Lopez et al. 2004) and animal (Pacher et al. 2003) studies involving unspecific NOS inhibitors. 
Acknowledgements The authors thank Dr. Kurt Reifenberg and other employees from the animal facility of University Mainz for excellent support. This work was supported by the German Nationales Genomforschungsnetz 2 (NGFN2) grant 01GS0421.

\section{References}

Barouch LA, Harrison RW, Skaf MW, Rosas GO, Cappola TP, Kobeissi ZA, Hobai IA, Lemmon CA, Burnett AL, O'Rourke B, Rodriguez ER, Huang PL, Lima JA, Berkowitz DE, Hare JM (2002) Nitric oxide regulates the heart by spatial confinement of nitric oxide synthase isoforms. Nature 416:337-339

Brahmajothi MV, Campbell DL (1999) Heterogeneous basal expression of nitric oxide synthase and superoxide dismutase isoforms in mammalian heart: implications for mechanisms governing indirect and direct nitric oxide-related effects. Circ Res 85:575-587

Brunner F, Andrew P, Wolkart G, Zechner R, Mayer B (2001) Myocardial contractile function and heart rate in mice with myocyte-specific overexpression of endothelial nitric oxide synthase. Circulation 104:3097-3102

Chaiswing L, Cole MP, Ittarat W, Szweda LI, St Clair DK, Oberley TD (2005) Manganese superoxide dismutase and inducible nitric oxide synthase modify early oxidative events in acute adriamycin-induced mitochondrial toxicity. Mol Cancer Ther 4:1056-1064

Cole MP, Chaiswing L, Oberley TD, Edelmann SE, Piascik MT, Lin SM, Kiningham KK, St Clair DK (2006) The protective roles of nitric oxide and superoxide dismutase in adriamycin-induced cardiotoxicity. Cardiovasc Res 69:186-197

Doroshow JH, Locker GY, Myers CE (1980) Enzymatic defenses of the mouse heart against reactive oxygen metabolites: alterations produced by doxorubicin. J Clin Invest 65:128-135

Duquaine D, Hirsch GA, Chakrabarti A, Han Z, Kehrer C, Brook R, Joseph J, Schott A, Kalyanaraman B, Vasquez-Vivar J, Rajagopalan S (2003) Rapid-onset endothelial dysfunction with adriamycin: evidence for a dysfunctional nitric oxide synthase. Vasc Med 8:101-107

Fogli S, Nieri P, Breschi MC (2004) The role of nitric oxide in anthracycline toxicity and prospects for pharmacologic prevention of cardiac damage. Faseb J 18:664-675

Garner AP, Paine MJ, Rodriguez-Crespo I, Chinje EC, Ortiz De Montellano P, Stratford IJ, Tew DG, Wolf CR (1999) Nitric oxide synthases catalyze the activation of redox cycling and bioreductive anticancer agents. Cancer Res 59:1929-1934

Gewirtz DA (1999) A critical evaluation of the mechanisms of action proposed for the antitumor effects of the anthracycline antibiotics adriamycin and daunorubicin. Biochem Pharmacol $57: 727-741$

Huang PL, Dawson TM, Bredt DS, Snyder SH, Fishman MC (1993) Targeted disruption of the neuronal nitric oxide synthase gene. Cell 75:1273-1286

Joshi P, Vig PJ, Veerisetty V, Cameron JA, Sekhon BS, Desaiah D (1996) Increase in brain nitric oxide synthase activity in daunorubicin-treated rats. Pharmacol Toxicol 78:99-103

Kalivendi SV, Kotamraju S, Zhao H, Joseph J, Kalyanaraman B (2001) Doxorubicin-induced apoptosis is associated with increased transcription of endothelial nitric-oxide synthase. Effect of antiapoptotic antioxidants and calcium. J Biol Chem 276:47266-47276

Kassner N, Huse K, Martin HJ, Godtel-Armbrust U, Metzger A, Meineke I, Brockmoller J, Klein K, Zanger UM, Maser E, Wojnowski L (2008) Carbonyl reductase 1 is a predominant doxorubicin reductase in the human liver. Drug Metab Dispos $36: 2113-2120$

Laubach VE, Shesely EG, Smithies O, Sherman PA (1995) Mice lacking inducible nitric oxide synthase are not resistant to lipopolysaccharide-induced death. Proc Natl Acad Sci U S A 92:10688-10692

Li T, Singal PK (2000) Adriamycin-induced early changes in myocardial antioxidant enzymes and their modulation by probucol. Circulation 102:2105-2110

Li T, Danelisen I, Bello-Klein A, Singal PK (2000) Effects of probucol on changes of antioxidant enzymes in adriamycininduced cardiomyopathy in rats. Cardiovasc Res 46:523-530

Liao Y, Takashima S, Zhao H, Asano Y, Shintani Y, Minamino T, Kim J, Fujita M, Hori M, Kitakaze M (2006) Control of plasma glucose with alpha-glucosidase inhibitor attenuates oxidative stress and slows the progression of heart failure in mice. Cardiovasc Res 70:107-116

Lopez A, Lorente JA, Steingrub J, Bakker J, McLuckie A, Willatts S, Brockway M, Anzueto A, Holzapfel L, Breen D, Silverman MS, Takala J, Donaldson J, Arneson C, Grove G, Grossman S, Grover R (2004) Multiple-center, randomized, placebo-controlled, double-blind study of the nitric oxide synthase inhibitor 546C88: effect on survival in patients with septic shock. Crit Care Med 32:21-30

Luo D, Vincent SR (1994) Inhibition of nitric oxide synthase by antineoplastic anthracyclines. Biochem Pharmacol 47:2111-2112

Lyu YL, Kerrigan JE, Lin CP, Azarova AM, Tsai YC, Ban Y, Liu LF (2007) Topoisomerase II \{beta\} mediated DNA double-strand breaks: implications in doxorubicin cardiotoxicity and prevention by dexrazoxane. Cancer Res 67:8839-8846

Minotti G, Menna P, Salvatorelli E, Cairo G, Gianni L (2004) Anthracyclines: molecular advances and pharmacologic developments in antitumor activity and cardiotoxicity. Pharmacol Rev $56: 185-229$

Mungrue IN, Husain M, Stewart DJ (2002) The role of NOS in heart failure: lessons from murine genetic models. Heart Fail Rev 7:407-422

Neilan TG, Blake SL, Ichinose F, Raher MJ, Buys ES, Jassal DS, Furutani E, Perez-Sanz TM, Graveline A, Janssens SP, Picard MH, Scherrer-Crosbie M, Bloch KD (2007) Disruption of nitric oxide synthase 3 protects against the cardiac injury, dysfunction, and mortality induced by doxorubicin. Circulation 116:506-514

Pacher P, Liaudet L, Bai P, Mabley JG, Kaminski PM, Virag L, Deb A, Szabo E, Ungvari Z, Wolin MS, Groves JT, Szabo C (2003) Potent metalloporphyrin peroxynitrite decomposition catalyst protects against the development of doxorubicin-induced cardiac dysfunction. Circulation 107:896-904

Sanz MJ, Hickey MJ, Johnston B, McCafferty DM, Raharjo E, Huang PL, Kubes P (2001) Neuronal nitric oxide synthase (NOS) regulates leukocyte-endothelial cell interactions in endothelial NOS deficient mice. Br J Pharmacol 134:305-312

Shesely EG, Maeda N, Kim HS, Desai KM, Krege JH, Laubach VE, Sherman PA, Sessa WC, Smithies O (1996) Elevated blood pressures in mice lacking endothelial nitric oxide synthase. Proc Natl Acad Sci U S A 93:13176-13181

Talman WT, Nitschke Dragon D (2007) Neuronal nitric oxide mediates cerebral vasodilatation during acute hypertension. Brain Res 1139:126-132

van Dalen EC, Caron HN, Dickinson HO, Kremer LC (2005) Cardioprotective interventions for cancer patients receiving anthracyclines. Cochrane Database Syst Rev CD003917

van Dalen EC, van der Pal HJ, Kok WE, Caron HN, Kremer LC (2006) Clinical heart failure in a cohort of children treated with anthracyclines: a long-term follow-up study. Eur J Cancer 42:3191-3198 
Vasquez-Vivar J, Martasek P, Hogg N, Masters BS, Pritchard KA Jr, Kalyanaraman B (1997) Endothelial nitric oxide synthasedependent superoxide generation from adriamycin. Biochemistry 36:11293-11297

Von Hoff DD, Layard MW, Basa P, Davis HL Jr, Von Hoff AL, Rozencweig M, Muggia FM (1979) Risk factors for doxorubicininduced congestive heart failure. Ann Intern Med 91:710-717
Weinstein DM, Mihm MJ, Bauer JA (2000) Cardiac peroxynitrite formation and left ventricular dysfunction following doxorubicin treatment in mice. J Pharmacol Exp Ther 294:396-401

Yen HC, Oberley TD, Vichitbandha S, Ho YS, St Clair DK (1996) The protective role of manganese superoxide dismutase against adriamycin-induced acute cardiac toxicity in transgenic mice. J Clin Invest 98:1253-1260 